\title{
On Fixed Point Theorem in Fuzzy Normed Space
}

\author{
Rana A. Mohammed*, Buthainah A. A. Ahmed* and Fadhel F. S. ${ }^{* *}$ \\ * Department of Mathematics, College of Science, Baghdad University, Baghdad-Iraq. \\ ${ }^{* * *}$ Department of Mathematics and Computer Applications, College of Science, \\ Al-Nahrain University, Baghdad-Iraq.
}

\begin{abstract}
The formal balls in fuzzy normed space $X$ (characterized by closed balls in $X$ ) are ordered by reverse inclusion depending on the concept of level sets. The set of formal balls in a fuzzy normed space is called a fuzzy domain normed space denoted by $B X$. This set is directed complete partially ordered set (dcpo), its maximal elements are the suprema. A contraction mapping principle is defined on $B X$. Banach fixed point theorem is studied and proved on $B X$.
\end{abstract}

Keywords: Fuzzy domain, dcpo, Formal balls, fixed point in fuzzy dcpo.

\section{Introduction}

The domain theory is that branch of mathematics which deals with some class of ordered sets. more specifically, it deals with the ordered sets Possessing suprema, or optimally are called domains. Domain theory is proposed by D. Scott [13] to introduce some computational models for computer engineering.it is employed to construct a mathematical framework for some programming languages. Abramsky in 1994 modified and reformulate the topic to have the contemporary structure, while fuzzy sets are simulating the real life more than crisp sets influence as it is known [1].

When Zadeh [15] introduced the concept of fuzzy sets, many contributions added in different mathematical subjects. The fuzzy sets were used widely in functional analysis, and many authors enriched the matter, like Kramosil [10], George and Veermani [6] are constructing the fuzzy metric spaces, Katras [9], Bag and Samantha [3] introduced and modified concept of fuzzy normed space, Goguen [8], and Sanchez [12] defined and studied fuzzy relations. Fuzzy partial ordered relations are introduced by Chon [4], while Yuan and Wu [14] introduced the concept of sub lattice. Adalat and Heckmann in 1998 connected the subject of domain theory with metric spaces and they obtained a computational model for metric space. The fuzzy normed spaces with domain theory are used as mathematical tools to construct the concept of fuzzy domain normed space [11], and so many articles added to the subject later.
This paper displays the abstract analysis for the fuzzy domain normed space. It defines the concept of monotone, Scott continuous, and contraction mappings.

In order to continue the research and study of the analysis for the fuzzy partially ordered sets (fuzzy domain theory), this paper submitted the maximal elements of directed sub sets of $B X$ as the suprema, these are employed to be limit points of convergent sequences of $B X$, finally these limit points Played an important role to obtain and prove the fixed point theorem in the complete fuzzy normed space that called fuzzy Banach domain space.

\section{Preliminaries}

This section presents some elementary and basic concepts related to this article.

\section{Definition (1), [1]:}

A non-empty set $P$ with a binary relation $\sqsubseteq$ is called partially ordered set or (poset) denoted by $(P, \subseteq)$, if the following holds for all $x, y, z \in P$ :

1. $x \subseteq x \quad$ (Reflexivity).

2. $x \sqsubseteq y \wedge y \sqsubseteq z \Rightarrow x \subseteq z$ (Transitivity).

3. $x$ 돼 $y$ 드 $x \Rightarrow x=y$ (Antisymmetry)

The ordered pair $(P, \sqsupseteq)$ is a poset too. when $\sqsupseteq$ is the reverse relation of $\sqsubseteq$. An element $x \in$ $P$ is said to be maximal element of $P$ if for all $y \in P$ such that $x \sqsubseteq y$, implies $x=y$. The dual concept for minimal element can be defined by the same way. 
Let $Y \subseteq P$, then the greatest element of $Y$ is an element $z \in Y$ such that $y \sqsubseteq z$ for all $y \in Y$. A top element of $P$ is the greatest element of $P$. An upper bond of $Y$ is an element $x \in P$ in which $y \subseteq x$ for all $y \in$ $Y$. The supremum of $Y$ is the unique element $x \in P$ which is the least element of the set of upper bounds of $P$. If the least upper bound is found, it will be denoted by $\sqcup Y$. Also, $Y$ will be defined as a directed set if each pair of elements of $Y$ has supremum. An ordered set $(P, \sqsubseteq$ ) is said to be directed complete partially ordered set or, briefly dcpo or, domain if every directed subset of $P$ has supremum.

\section{Definition (2), [4]:}

Let $X$ be a non-empty set. A function $A: X \times X \rightarrow[0,1]$ is called a fuzzy relation in $X$. The fuzzy relation $A$ is called reflexive if and only if $A(x, x)=1, A$ is antisymmetry if and only if $A(x, y) \geq 0, A(y, x) \geq 0$ implies $x=y$, and $A$ is transitive if and only if $A(x, z) \geq \sup _{y \in X} \min ((A(x, y), A(y, z))$, for all $x, y, z \in X$. A fuzzy relation $A$ is said to be fuzzy partial ordered relation if $A$ is reflexive, antisymmetric, and transitive. A fuzzy relation is totally ordered if and only if $A(x, y) \geq 0$ or $A(y, x) \geq 0$ for all $x, y \in X$. If $A$ is a fuzzy partial ordered relation in $X$ then the ordered pair $(X, A)$ is said to be a fuzzy partial ordered set or a fuzzy poset, moreover if $A$ is a totally ordered then $(X, A)$ is said to be a fuzzy totally ordered set or a fuzzy chain.

\section{Definition.(3), [6]:}

A binary operation $*:[0,1] \times[0,1] \rightarrow[0,1]$ is a continuous t-norm if it satisfies the following conditions.

1. * is associative and commutative;

2. $*$ is continuous;

3. $a * 1=a$ for all $a \in[0,1]$;

4. $a * b \leq c * d$ whenever $a \leq c$ and $b \leq d$, for each $a, b, c, d \in[0,1]$. Two typical examples of continuous t-norm are $a * b=a b$ and $a * b=\min (a, b)$. This paper will restrict the research on the minimum t-norm binary operation.

\section{Definition (4), [6]:}

The third tuple $(X, M, *)$ is called a fuzzy metric space if $X$ is a non-empty set, $*$ is a continuous t-norm, and $M$ is a fuzzy set on
$X^{2} \times(0, \infty)$, satisfying the following conditions for each $x, y, z \in X$ and $t, s>0$,

1. $M(x, y, t)>0$

2. $M(x, y, t)=1$ if and only if $x=y$;

3. $M(x, y, t)=M(y, x, t)$,

4. $(x, y, t) * M(y, z, s) \leq M(x, z, t+s)$;

5. $M(x, y,):.(0, \infty) \rightarrow[0,1]$

is continuous.

\section{Definition (5), [3]:}

Let $X$ be a linear space over a field of scalars $\mathbb{F}$. A fuzzy subset $N$ of $X \times \mathbb{R}$ is called a fuzzy norm on $X$ if $\forall x, y \in X$ and $c \in \mathbb{F}$,

1. $\forall t \in \mathbb{R}$ with $t \leq 0 ; N(x, t)=0$,

2. $\forall t \in \mathbb{R}$ with $t>0 ; N(x, t)=$

1 if and only if $x=0$;

3. $\forall t \in \mathbb{R}$ with $t>0 ; N(c x, t)=$ $N(x, t /|c|)$ if $c \neq 0$;

4. $\forall s, t \in \mathbb{R}, x, y \in X ; N(x+y, s+t) \geq$ $\min \{N(x, s), N(x, t)\}$

5. $N(x, \cdot)$ is a non-decreasing function of $\mathbb{R}$ and $\lim _{t \rightarrow \infty} N(x, t)=1$.

The pair $(X, N)$ will be referred to as a fuzzy normed linear space.

\section{Note:}

The relation $\min \{N(x, s), N(y, t)\}$ will be replaced by $N(x, s) * N(y, t)$ and the fuzzy normed space will be denoted by $(X, N, *)$.

\section{Definition (6), [11]:}

Let $X$ be a fuzzy normed space with closed balls defined by:

$$
B[x, \alpha, r]=\{y \in X \mid N(x, r) \geq \alpha \forall r \geq 0\}
$$

Closed balls in $X$ will be denoted by $[\mathrm{x}, \alpha, \mathrm{r}]$ and arranged by reverse inclusion denoted by $\sqsubseteq$.

\section{Definition (7), [11]:}

A formal ball in a fuzzy normed space $(X, N, *)$ is the ordered triple $[x, \alpha, r]$ where $x \in X, 0 \leq \alpha \leq 1$ and $r>0$ in which for every $y \in X, 0<\beta<\alpha<1, r>s>0$, one can define:

$$
\begin{aligned}
& {[x, \alpha, r] \subseteq[y, \beta, s] \text { if and only if }} \\
& N(x-y, r-s) \geq 1-(\alpha-\beta)
\end{aligned}
$$


The set of formal balls will be denoted by $B X$, the ordered pair $(B X, \sqsubseteq)$ will be called fuzzy domain normed space.

\section{Remark (1):}

The pair $(B X$, 드) is a fuzzy poset, actually $(B X$, 드) is fuzzy chain, (for more details one can see [11]). Studying the convergence and divergence among elements of $B X$ is as like as Studying the increasing, and decreasing of their cut sets, this similarity led to define the convergence in $B X$ as follows:

\section{Definition (8), [11]:}

Let $\left\{\left[x_{n}, \alpha_{n}, r_{n}\right]\right\}$ be a sequence of elements of $B X$ that ordered in the form:

$$
\begin{aligned}
& \left\{\left[x_{n}, \alpha_{n}, r_{n}\right]\right\} \subseteq\left\{\left[x_{n+1}, \alpha_{n+1}, r_{n+1}\right]\right\} \text { for all } \\
& \mathrm{n} \in \mathbb{N} \text {. }
\end{aligned}
$$

A sequence $\left\{\left[x_{n}, \alpha_{n}, r_{n}\right]\right\}$ is called Cauchy sequence in $B X$ if:

$$
\begin{aligned}
& \lim _{n \rightarrow \infty} N\left(x_{n}-x_{m}, r_{n}-r_{m}\right) \geq, \\
& 1-\left(\alpha_{n}-\alpha_{m}\right) \text { i.e., } x_{n}-x_{m} \rightarrow 0, r_{n}-r_{m}>0
\end{aligned}
$$$$
\text { and }\left\{\left[x_{n}, \alpha_{n}, r_{n}\right]\right\} \text { converges to }\{[y, \beta, s]\} \text { in } B X
$$
if:

$$
\lim _{n \rightarrow \infty} N\left(x_{n}-y, r_{n}-s\right) \geq 1-\left(\alpha_{n}-\beta\right) \text {, as }
$$

$n \rightarrow \infty$

then $\alpha_{n} \rightarrow 0$ which means $\left\{\left[x_{n}, \alpha_{n}, r_{n}\right]\right\}$ converges to $\{[y, 0, s]\}$.

\section{Lemma (1), [11]:}

According to the reverse inclusion the maximal elements of $B X$ can be considered to be contained in the set:

$$
U X=\{[x, 0, r] \mid x \in X, r>0\},
$$

against the set of closed balls with center $x$ and radius 0 for all $r>0$.

\section{Theorem (10), [1]:}

Let $(B X$, 드) be a fuzzy domain normed space, and $\left\{\left[x_{n}, \alpha_{n}, r_{n}\right]\right\}$ be an ascending sequence in $B X,[y, \beta, s]$ is an element of $B X$, then the following statements are equivalent:

1. $[y, \beta, s]$ is a least upper bound of $\left\{\left[x_{n}, \alpha_{n}, r_{n}\right]\right\}_{n \in \mathbb{N}}$.

2. $[y, \beta, s]$ is an upper bound of $\left\{\left[x_{n}, \alpha_{n}, r_{n}\right]\right\}_{n \in \mathbb{N}}$, with $\lim _{n \rightarrow \infty} \alpha_{n}=\beta$.

3. $\lim _{n \rightarrow \infty} x_{n}=y$ and $\lim _{n \rightarrow \infty} \alpha_{n}=\beta$, i. e. $x_{n}-y \rightarrow 0, \alpha_{n}-\beta \rightarrow 0$, as $n \rightarrow \infty$

\section{Lemma (2):}

$B X$ is a dcpo if and only if each sequence of $B X$ converges to its maximal element.

\section{Proof:}

Let $\left\{\left[x_{n}, \alpha_{n}, r_{n}\right]\right\}$ be an ascending sequence in $B X$, as $n \rightarrow \infty$ then $\alpha_{n} \rightarrow 0$, so it is enough to prove that $\left[x_{n}, \alpha_{n}, r_{n}\right] \rightarrow[y, 0, s]$ if and only if $[y, 0, s]$ is a least upper bound of $\left\{\left[x_{n}, \alpha_{n}, r_{n}\right]\right\}$

$\Rightarrow$ ) Suppose that $[y, 0, s]$ is a limit point of $\left\{\left[x_{n}, \alpha_{n}, r_{n}\right]\right\}$, then by definition (6)

$$
\begin{aligned}
& \lim _{n \rightarrow \infty} N\left(x_{n}-y, r_{n}-s\right) \geq 1-\left(\alpha_{n}-0\right) \\
& \text { Then } \lim _{n \rightarrow \infty} N\left(x_{n}-y, r_{n}-s\right)=1 \quad \text { and }
\end{aligned}
$$
therefore from definition (3) $x_{n}-y \rightarrow 0$, which yields:

$x_{n} \rightarrow y$, and $\alpha_{n} \rightarrow 0$ as $n \rightarrow \infty$, then by theorem (1), $[y, 0, s]$ is a least upper bound of the sequence

$\Longleftarrow)$ Suppose that $[y, 0, s]$ is aleast upper bound of $\left\{\left[x_{n}, \alpha_{n}, r_{n}\right]\right\}$ then by theorem (1) $x_{n} \rightarrow y$, hence $x_{n}-y \rightarrow 0, \alpha_{n} \rightarrow 0$, then:

$$
\lim _{n \rightarrow \infty} N\left(x_{n}-y, r_{n}-s\right)=1
$$

Hence $\left\{\left[x_{n}, \alpha_{n}, r_{n}\right]\right\}$ converges to $[y, 0, s]$ by theorem (1).

\section{Remark (2):}

The above lemma introduced an important fact represented by joining concepts in domain theory and compares them in fuzzy normed spaces and domains defined on it is worth mentioning here, that is a fuzzy domain normed space is complete if and only if it is a dcpo.

\section{T-Banach contraction principle of fuzzy domain normed spaces}

First of all we must mention some elementariness in the subject of fuzzy metric spaces, and fuzzy domain normed spaces to obtain full use of the results.

\section{Definition (9), [1]:}

Let $P$ and $Q$ be partially ordered sets.A function $f: P \rightarrow Q$ is called monotone if for all $x, y \in P$ with $x \sqsubseteq y$ then $f(x) \sqsubseteq f(y)$ in $Q$. If each of $P$ and $Q$ are dcpo then a monotone function $f: P \rightarrow Q$ is said to be 
Scott continuous if $f(\sqcup A)=\sqcup f(A)$ for any directed subset A of $P$.

Definition (10), [5]:

Let $(X, M, *)$ be a fuzzy metric space. A mapping $T: X \rightarrow X$ is said to be sequentially convergent if we have for every sequence $\left\{y_{n}\right\}$; if $\left\{\mathrm{Ty}_{\mathrm{n}}\right\}$ is convergent then $\left\{y_{n}\right\}$ is convergent.

\section{Definition (11), [5]:}

Let $(X, M, *)$ be a fuzzy metric space.A mapping $T: X \rightarrow X$ is said to be sub sequentially convergent if we have for every sequence $\left\{y_{n}\right\}$, if $\left\{\mathrm{Ty}_{n}\right\}$ is convergent then $\left\{y_{n}\right\}$ has convergent subsequence.

\section{Definition(12), [5]:}

Let $(X, M, *)$ be a fuzzy metric space. and let $T, S: X \rightarrow X$ be any two self-mappings.

A mapping $S$ is said to be fuzzy $T$ contraction if there exists $k \in(0,1)$ such that $M(T S x, T S y, k t) \geq M(T x, T y, t)$, for all $x, y \in$ $X$ and $t>0$. If $T$ is the identity mapping then then $S$ is fuzzy contraction.

\section{Theorem (2), [5]:}

Let $(X, M, *)$ be a complete fuzzy metric space and $T: X \rightarrow X$ be a continuous one to one and sub sequentially convergent mapping. Let $M(x, y, t) \rightarrow 1$ as $t \rightarrow \infty$, For all $x, y \in$ $X$. Then a fuzzy $T$ - contraction mapping $S: X \rightarrow X$ has a unique fixed point, also if $T$ is a sequentially convergent, then for each $x_{0} \in$ $X$, the sequence of iterates $\left\{S^{n} x_{0}\right\}$ converges to that fixed point.

The main results obtained below, as $T$ is the identity mapping.

\section{Contraction mapping principle in Fuzzy Domain normed spaces}

As is known, the contraction mapping are defined on metric spaces now we will try to find a way to define such mappings on the dcpos defined in the fuzzy normed spaces.as follows.

\section{Definition (13):}

Let $S: X \rightarrow Y$ be a mapping between two fuzzy normed spaces $X, Y$. A Lipchitz constant for $S$ is a number $k \in \mathbb{R}^{+}$, such that for all $x, x_{0} \in X \quad N\left(S x-S x_{0}, k t\right) \geq N\left(x-x_{0}, t\right)$, holds. If $k \in(0,1)$, then $S$ is said to be contraction mapping. The collection pairs $(s, k)$ of mappings with their Lipchitz constants form a category with $i d_{X}=\left(i d_{X}, 1\right)$ and $(S, k) \circ\left(S_{0}, k_{0}\right)=\left(S \circ S_{0}, k k_{0}\right)$, the functor $B$ defined on this category defined by $B(S, k)[x, \alpha, r]=[S x, \alpha, k r]$ for every $[x, \alpha, r] \in B$. Moreover

$[S x, \alpha, k r] \subseteq\left[T x, \beta, k_{0} s\right]$ defined by $N\left(S x-T y, k r-k_{o} s\right) \geq 1-(\alpha-\beta)$

\section{Definition (14):}

Let $B X$ be a fuzzy domain normed space. A mapping $g: B X \rightarrow B X$ is defined by $g[x, \alpha, r]=B(S, k)[x, \alpha, r]=[S x, \alpha, k r]$. The mapping $g$ is said to be monotone if whenever $[x, \alpha, r] \sqsubseteq[y, \beta, s]$ then $g[x, \alpha, r] \sqsubseteq g[y, \beta, s]$, and if $B X$ is complete then $\mathrm{g}$ is said to be scott continuous if for any subset $A$ of $B X$ then $\sup (g(A))=g(\sup (A))$.

\section{Lemma (3):}

If $S$ is contraction then $g$ is monotone and scott continuous.

\section{Proof:}

Suppose that $[x, \alpha, r] \sqsubseteq[y, \beta, s]$ for some $x, y \in X, s, t>0$ and $\alpha, \beta \in(0,1)$.

Since $S$ is contraction then:

$$
\begin{aligned}
N(S x-S y, k r-k s) & \geq N(x-y, r-s) \\
& \geq 1-(\alpha-\beta)
\end{aligned}
$$

Then $[S x, \alpha, k r] \subseteq[S y, \beta, k s]$ that is $g[x, \alpha, r] \sqsubseteq g[y, \beta, s]$ and hence $g$ is monotone

To show that $g$ is scott continuous let $\left\{\left[x_{n}, \alpha_{n}, r_{n}\right]\right\}$ be an ascending sequence of elements of $B X$ with least upper bound $[y, 0, s]$. "since $B X$ is a dcpo" it is known that $S$ is contraction then as it will be seen above $g$ is monotone then the sequence $\left\{g\left[x_{n}, \alpha_{n}, r_{n}\right]\right\}$ is an ascending too, it is enough to show that $\sup \left\{g\left[x_{n}, \alpha_{n}, r_{n}\right]\right\}=g\left(\sup \left\{\left[x_{n}, \alpha_{n}, r_{n}\right]\right\}\right)$.

Since $[y, 0, s]$ is a least upper bound of $\left\{\left[x_{n}, \alpha_{n}, r_{n}\right]\right\}$ then by theorem (1), $\lim _{n \rightarrow \infty} x_{n}=y$ and, $S$ is continuous hence $\lim _{n \rightarrow \infty} S x_{n}=S y$, as well as $\lim _{n \rightarrow \infty} \alpha_{n}=0$ then, then by theorem (1) $[S y, 0, k s]$ is aleast upper bound for the ascending sequence $\left\{\left[S x_{n}, \alpha_{n}, k^{n} r_{n}\right]\right\}$ then we done. 
The main result in this paper will be represented below by introduced the concept of fixed point in fuzzy domain Banach space.

\section{Definition (15):}

Let $B X$ be a fuzzy domain normed space. A self-mapping $g: B X \rightarrow B X$ is said to have a fixed point if there exists some element $[x, \alpha, r]$ in $B X$ such that $g[x, \alpha, r]=[x, \alpha, r]$.

\section{Theorem (3):}

Let $(X, M, *)$ be a fuzzy Banach space $\mathrm{A}$ mapping $S: X \rightarrow X$ is contraction, then $g: B X \rightarrow B X$ with $g[x, \alpha, r]=[S x, \alpha, k r]$ has a unique fixed point. Moreover the sequence $\left\{S^{n}\left(x_{0}\right)\right\}$ of iterates converges to that fixed point.

\section{Proof:}

Define the orbit $\left\{S^{n}\left(x_{0}\right)\right\}$ by: $x_{n+1}=$ $S\left(x_{n}\right), x_{n}=S\left(x_{n-1}\right), \ldots x_{1}=S\left(x_{0}\right)$.

For any ascending sequence $\left\{\left[x_{n}, \alpha_{n}, r_{n}\right]\right\}$ in $B X$ the sequence:

$$
g\left(\left\{\left[x_{n}, \alpha_{n}, r_{n}\right]\right\}\right)=\left\{\left[S x_{n}, \alpha_{n}, k^{n} r_{n}\right]\right\}
$$

is an ascending sequence since $g$ is monotone, that is $\left[S x_{n-1}, \alpha_{n-1}, k^{n-1} r\right] \sqsubseteq$ $\left[S x_{n}, \alpha_{n}, k^{n} r_{n}\right]$ for each $n>1$, then one can obtain that:

$$
\begin{aligned}
N\left(x_{n+1}\right. & \left.-x_{n}, k t\right)=N\left(S x_{n}-S x_{n-1}, k t\right) \\
& \geq N\left(x_{n}-x_{n-1}, t\right) \\
& =N\left(S x_{n-1}-S x_{n-2}, t\right) \\
& \geq N\left(x_{n-1}-x_{n-2}, \frac{t}{k}\right) \\
& (\text { since } S \text { is contraction }) \\
& =N\left(S x_{n-2}-S x_{n-3}, \frac{t}{k}\right) \\
& \geq N\left(x_{n-2}-x_{n-3}, \frac{t}{k^{2}}\right) \\
& \geq N\left(x_{1}-x_{0}, \frac{t}{k^{n-1}}\right) \ldots \ldots \ldots \ldots \ldots . . .
\end{aligned}
$$

Hence for any integer $p$ (with definition of fuzzy norm and properties of minimum binary operation $*$ ), one can conclude that:

$$
\begin{aligned}
& N\left(x_{n}-x_{n+p}, t\right) \geq N\left(x_{n}-x_{n+1}, \frac{t}{p}\right) * \\
& N\left(x_{n+1}-x_{n+p}, \frac{(p-1) t}{p}\right) \\
& \geq N\left(x_{n}-x_{n+1}, \frac{t}{p}\right) *\left[N\left(x_{n+1}-x_{n+2}, \frac{t}{p}\right) *\right. \\
& \left.N\left(x_{n+2}-x_{n+p}, \frac{(p-2) t}{p}\right)\right]
\end{aligned}
$$

Till the term $(p-1)$

$$
\begin{aligned}
& =N\left(x_{n}-x_{n+1}, \frac{t}{p}\right) * N\left(x_{n+1}-x_{n+2}, \frac{t}{p}\right) * \ldots * \\
& N\left(x_{n+p-1}-x_{n+p}, \frac{t}{p}\right) \\
& \geq N\left(x_{1}-x_{0}, \frac{t}{k^{n} p}\right) * N\left(x_{1}-x_{0}, \frac{t}{k^{n+1} p}\right) * \ldots * \\
& N\left(x_{1}-x_{0}, \frac{t}{k^{n+p-1} p}\right)(\text { from }(1))
\end{aligned}
$$

As $n \rightarrow \infty$ then each term approaches 1 hence

$$
N\left(x_{n}-x_{n+p}, t\right) \geq 1 * 1 * \ldots * 1=1
$$

Then $\lim _{n \rightarrow \infty} N\left(x_{n}-x_{n+p}, t\right)=1$, then $\left\{\left[S x_{n}, \alpha_{n}, k^{n} r_{n}\right]\right\}$ is Cauchy sequence, since $B X$ is a dcpo then from lemma (2) $\left\{\left[S x_{n}, \alpha_{n}, k^{n} r\right]\right\}$ is convergent to its least upper bound and to compute it let us consider the ascending sequence $\left\{\left[x_{n}, \alpha_{n}, r_{n}\right]\right\}$ with least upper bound represented by maximal element $[y, 0, s]$.

Since $g$ is Scott continuous then:

$$
\sup \left\{g\left[x_{n}, \alpha_{n}, r_{n}\right]\right\}=g\left(\sup \left\{\left[x_{n}, \alpha_{n}, r_{n}\right]\right\}\right)
$$

and $g[y, 0, s]=\sup \left(\left\{\left[S^{n} x_{0}, \alpha_{n}, k^{n} r_{n}\right]\right\}\right)$ then, $\{[S y, 0, k s]\}=\sup \left(\left\{\left[S^{n} x_{0}, \alpha_{n}, k^{n} r_{n}\right]\right\}\right)$

Hence from lemma (3) one obtained that $\lim _{n \rightarrow \infty}\left[S^{n} x_{0}, \alpha_{n}, k^{n} r_{n}\right]=[S y, 0, k s]$

From theorem (1) we have that $\lim _{n \rightarrow \infty} S^{n} x_{0}=$ $S y$ then $\lim _{n \rightarrow \infty} S^{n-1} x_{0}=y$, and $\lim _{n \rightarrow \infty} \alpha_{n}=0$, that leads to conclude that $[y, 0, s]$ is a least upper bound of $\left\{\left[S^{n} x_{0}, \alpha_{n}, k^{n} r_{n}\right]\right\}$ but the limit point is unique then $[S y, 0, k s]=[y, 0, s]$ in other wards $g[y, 0, s]=[y, 0, s]$ that is $[y, 0, s]$ is a fixed point.

Now to prove the uniqueness of the fixed point Suppose that $[z, \gamma, t]$ is another fixed point of $g$ then either $[y, 0, s] \sqsubseteq[z, \gamma, t]$ or $[z, \gamma, t] \subseteq[y, 0, s]$ if $[y, 0, s] \subseteq[z, \gamma, t]$, then: $N(y-z, s-t) \geq 1-(-\gamma)=1+\gamma$

Therefore, $\gamma$ must be 0 hence $[z, \gamma, t]$ is maximal element of $\left\{\left[x_{n}, \alpha_{n}, r_{n}\right]\right\}$ and by uniqueness of least upper bound, then $[z, \gamma, t]=[y, 0, s]$.

If $[z, \gamma, t] \subseteq[y, 0, s]$, then $[z, \gamma, t]$ is a least upper bound of $\left\{\left[x_{n}, \alpha_{n}, r_{n}\right]\right\}$ this yields to $[z, \gamma, t]=[y, 0, s]$.

\section{References}

[1] Abramsky S. and Jung A., "Domain theory", Ins. Abramsky, D. M. Gabbey and T. S. E. Maibaum (Eds.), Calrendon Press, Oxford, Vol.1, 1-168, 1994. 
[2] Adalat A. and Heckmann R., "A computational model for metric spaces", Theoret. Comput. Sci, 193, 53-73, 1998.

[3] Bag T. and Samanta S.K., "Finite dimensional fuzzy normed linear spaces", J. Fuzzy Math., Vol.1, No.3, 687-705, 2003.

[4] Chon, Fuzzy I., "Partial ordered Sets and Lattices", Korean J. Math. 17 No. 4, 3613742009.

[5] Dey D. and Saha M., "An extension of Banach fixed point theorem in fuzzy metric spaces" Bol. Soc. Mat., Vol. 32, No. 1, 301306, 2014.

[6] George A. and Veermani, "On some results in fuzzy metric spaces", Fuzzy Sets and Systems, 64, 395-399, 1994.

[7] Grabiec M., "Fixed points in fuzzy metric space", Fuzzy Sets and Systems, 27, 385389, 1988.

[8] Goguen J. A., "L-fuzzy sets", J. Math. Anal. Appl. 18, 145-174, 1967.

[9] Katsaras A.K., "Fuzzy topological vector spaces", Fuzzy Sets and Systems, 12, 143154, 1984.

[10]Kramosil I., and Michalek J., "Fuzzy metric and statistical metric space", Kybernetica, 11, 326-334, 1975.

[11]Rana A. Mohammed, Buthainah A. A. Ahmed and Fadhel F. S, "On Completeness of Fuzzy Normed Spaces", Journal of Advanced in mathematics vol.9, No.8, 2963-2970, 2015.

[12] Sanchez E., "Resolution of composite fuzzy relation equation", Inform. and Control, 30, 38-48, 1976.

[13] Scott D., "Outlines of mathematical theory of computations", $4^{\text {th }}$. Ann. Princcton conference on information science and systems, 169-176, 1970.

[14] Yuan B.O. and Wu W., "Fuzzy Ideals on a Distributive lattice", Fuzzy Sets and Systems, 35, 231-240, 1990.

[15] Zadeh L.A., "Fuzzy sets", Information and Control, 8(3), 338-353, 1965. 